\title{
¿UNA MISMA DEONTOLOGÍA PARA DISTINTAS CIENCIAS? REVISIÓN DE PAUTAS NACIONALES E INTERNACIONALES EN ÉTICA DE LA INVESTIGACIÓN CIENTÍFICA
}

\begin{abstract}
María Inés Winkler Müler ${ }^{1}$, Antonio Letelier Soto ${ }^{1}$
Resumen: Se realizó una revisión de normas jurídicas chilenas y códigos éticos internacionales asociados a la investigación científica con seres humanos, a través de una metodología mixta de análisis de contenido, con el propósito de identificar la presencia de vocablos asociados a la psicología, comprendida como una ciencia intermedia entre la biomedicina y las ciencias sociales. Se busca conocer las consideraciones éticas presentes en ambos tipos de normativas, para delimitar las diferencias entre aquellas asociadas a las ciencias biomédicas y las que corresponden a la psicología.

Los resultados muestran una escasa presencia de normas ligadas a las particularidades de la psicología y un limitado y pobre uso de sus conceptos, evidenciando que la bioética, mayoritariamente basada en los desarrollos de la biomedicina, no responde adecuadamente a las necesidades de resolución de conflictos éticos en el contexto investigativo de la ciencia psicológica.
\end{abstract}

Palabras clave: ética, bioética, investigación con seres humanos, psicología, ciencias sociales

\section{One same deontology for different sciences? Review of national and international guidelines in ethics of scientific research}

\begin{abstract}
A review of legal Chilean norms and international ethical codes about research involving human beings was carried out, through a mix methodology of content analysis, with the purpose of identifying the presence of words associated to psychology, as an intermediate science between biomedicine and social sciences. Finding ethical issues present in both types of norms was looked out to delimit the differences between those associated to biomedical sciences and those to psychology. Results show a scarce presence of norms linked to the particularities of psychology and a limited and poor use of concepts, demonstrating that bioethics, mainly based on biomedical developments, does not respond adequately to the needs for conflict resolution in the research context of psychology.
\end{abstract}

Key words: ethics, bioethics, research involving human beings, psychology, social sciences

\section{Uma mesma deontologia para distintas ciências? Revisão de pautas nacionais e internacionais em ética da investigação científica}

Resumo: Foi realizada uma revisão de normas jurídicas chilenas e códigos éticos internacionais associados à investigação científica com seres humanos, através de uma metodologia mista de análise de conteúdo, com o propósito de identificar a presença de vocábulos associados à psicologia, compreendida como uma ciência intermediária entre a biomedicina e as ciências sociais. Busca-se conhecer as consideraçóes éticas presentes em ambos os tipos de normativas, para delimitar as diferenças entre aquelas associadas às ciências biomédicas e as que correspondem à psicologia.

Os resultados mostram uma escassa presença de normas ligadas às particularidades da psicologia e um limitado e pobre uso de seus conceitos, evidenciando que a bioética, majoritariamente baseada nos desenvolvimentos da biomedicina, não responde adequadamente às necessidades de resolução de conflitos éticos no contexto investigativo da ciência psicológica.

Palavras-chave: ética, bioética, investigação com seres humanos, psicologia, ciências sociais

\footnotetext{
${ }^{1}$ Escuela de Psicología, Universidad de Santiago de Chile, Chile

Correspondencia: maria.winkler@usach.cl
} 


\section{Introducción}

Una revisión somera de las normativas nacionales e internacionales que regulan los aspectos éticos de la investigación científica en seres humanos revela la carencia de pautas éticas específicas y normas jurídicas que definan el quehacer científico en el ámbito de las ciencias sociales. La mayoría de los lineamientos seguidos por investigadores/ as en ciencias sociales se circunscriben a normas, tratados y pautas éticas internacionales con criterios generales que regulan la investigación en biomedicina.

Gran parte de los problemas éticos de la ciencia han sido subsumidos por las ciencias tradicionales y sus sistemas de reflexión ética, particularmente por la bioética en el contexto de la biomedicina. No obstante, muchos son los cuestionamientos que surgen a partir del protagonismo de la bioética y su comprensión de la realidad científica. El que nos parece más acuciante es la aparente carencia de reflexión ética en torno a las ciencias sociales y humanas, teniendo en cuenta que sus métodos se diferencian de los utilizados por las ciencias biomédicas.

Considerando que la investigación en ciencias sociales constituye una dimensión relevante de la producción mundial y nacional, además de poseer fundamentos, intervenciones y productos sustantivamente diferentes de la investigación biomédi$\mathrm{ca}$, nos parece fundamental revisar los principios y normas que guían este tipo de investigaciones, en particular cuando se desarrollan en el trabajo con seres humanos.

El principal objetivo del presente trabajo es identificar la presencia - o ausencia- de vocablos referidos a temáticas propias de una ciencia que, para estos efectos, consideraremos social —la psicología- a través del análisis de un conjunto de normativas éticas y legales, nacionales e internacionales, que regulan la investigación científica.

\section{La investigación científica en seres humanos}

La participación de seres humanos en investigaciones científicas ha sido considerada una cuestión controversial, que produce variadas situaciones dilemáticas debatidas con creciente frecuencia en el mundo entero(1).
Las transgresiones a los derechos humanos cometidas en la experimentación con personas(2-4) han incidido en el interés por generar un marco regulador extensivo, considerando las declaraciones que consagran los derechos humanos y protegen la integridad física y psíquica de los sujetos de investigación(5).

Con este propósito, han sido generados dispositivos de evaluación ética y científica que asumen cuotas crecientes de responsabilidad en el seguimiento de los proyectos de investigación, buscando integrarse coherentemente con normas de diferente naturaleza (ética y/o jurídica), en sus distintos niveles y jurisdicciones. La distinción entre la reflexión ética y el alcance de la norma jurídica es necesaria en función de los ámbitos diferentes de que se trata, tanto por intención como por ascendiente $\operatorname{social}(6)$.

Como señala Sotomayor(4), el propósito es velar por la correcta aplicación de las normas, los valores y los principios, consensuados local o internacionalmente, protegiendo al mismo tiempo la libertad de investigar y los derechos humanos de quienes participan en investigaciones científicas.

Muchas comunidades científicas, organizaciones políticas y comunidades civiles han comenzado a adquirir conciencia del impacto social que la ciencia puede tener en la vida de las personas $(7,8)$, sobre todo en las condiciones sociales actuales, que implican transformaciones políticas, económicas y tecnológicas globales y, por tanto, un aumento de la complejidad y las disyuntivas éticas producidas en el terreno científico(9).

Ya no bastan criterios de excelencia técnica, sino que se requiere la instauración progresiva de una comunidad moral, con normativas claras y de fácil acceso(8), que permita delimitar áreas de acción de la ciencia y, sobre todo, su responsabilidad social y el papel que le atañe a los/las científicos/as y a la sociedad en su conjunto $(10,11)$.

La ética no viene a ser una ańadidura de la ciencia, sino que forma parte de las labores de la investigación científica. No busca solamente aclarar los valores morales que implica la disciplina en su trasfondo, sino que sustenta la discusión pública, los diálogos que se establecen entre ciencia, industria y $\operatorname{sociedad}(11)$. 
Los avances de las comunicaciones y los cambios sociales, así como el aumento de la inversión en investigación, incrementan las cuotas de control que la ciencia obtiene a través del conocimiento, difuminando el límite de lo que se creía posible y llevándolo a un terreno que excede el contexto cultural local, ya no técnico sino social y ético. La libertad de investigación, reconocida en la Declaración Americana de los Derechos y Deberes del Hombre, de 1948, debe condecirse con la protección a las personas, incluso de generaciones futuras(4).

Diversos documentos definen los límites éticos en las investigaciones. Una de las propuestas más difundidas es la de Emmanuel(12), que ha inscrito en el discurso sobre la ética de la investigación la relevancia del valor social y científico, validez científica, justa selección de los sujetos, positiva relación riesgo/beneficio, evaluación independiente, consentimiento informado y respeto por la autonomía de los sujetos. Requisitos consensuados internacionalmente mediante discusiones que someten los temas a un diálogo que intersecta la ética, el derecho, las ciencias y las comunidades involucradas.

Cualquier propuesta ética contemporánea debería profundizar en el supuesto que los procesos científico-tecnológicos no deben ser descontextualizados del marco socioeconómico en que se insertan, y las respuestas éticas no han de desarrollarse por encima de las relaciones sociales imperantes(7). Aréchiga(13) concuerda en este aspecto, al plantear que la ética de la ciencia ha cambiado al incursionar en el mundo de la empresa y el gobierno, debiendo interactuar con los conjuntos sociales.

\section{¿Diferentes ciencias responden a una misma ética?}

Delimitar el campo de estudio de la investigación científica es una tarea que requiere distinguir, por una parte, entre las ciencias biomédicas, que utilizan los insumos de las tecnociencias aplicados al campo de la biología, y, por otra, las ciencias sociales, circunscritas al estudio de 'lo social', definido desde múltiples variables socioculturales.

Ambos campos científicos orientan su trabajo hacia objetos diversos, que ocasionalmente conver- gen en el ámbito de las políticas públicas de salud o en los espacios académicos universitarios. Sin embargo, en la mayoría de estas instituciones las regulaciones se basan en la bioética y su orientación biomédica.

En el caso del desarrollo de las ciencias sociales, el dispositivo tecnocrático impuesto por la dictadura en Chile desde 1973 desmanteló el trabajo realizado, desgastando el tejido conectivo entre las instituciones, las universidades y las comunidades y dejando a las ciencias sociales desprovistas de gran parte de su producción académica empíri$\mathrm{ca}(14)$.

El retorno a la democracia instaló una ciencia tributaria de las reformas económicas y políticas que la dictadura institucionalizó. Las políticas sociales buscaron no desestabilizar el modelo económico y coordinarse con los cambios globales.

En los años 90, las ciencias sociales resurgieron. El Estado incrementó su demanda de investigaciones y el campo volvió a adquirir legitimidad social(15). Sin embargo, las sociedades occidentales atravesaron crisis cuyas coordenadas fueron el hundimiento del socialismo y el fin de la bipolaridad de los bloques políticos y económicos. Una de las consecuencias fue el debilitamiento de los ideales y el resurgir de fundamentalismos y nacionalismos, siendo el ético uno de los planos más dañados por la posmodernidad(16).

Las ciencias sociales han buscado comprender los componentes subjetivos de conciencia e interpretación en un entramado social(17). Sin embargo, la ética de la investigación, en este ámbito, ha encontrado múltiples obstáculos en su desarrollo, que recaen en la dificultad para definir y delimitar los componentes que configuran la ciencia social en su conjunto. Incluso, se discute que 'lo social' pueda ser un objeto de estudio científico(18).

Por una parte, Sánchez Vidal(16) expresa que la lógica 'posmoderna' postula un desmantelamiento de la ética, al menos en su acepción de canon universal, lo que lleva precisamente a la necesidad de intensificar la reflexividad propia de la disciplina.

Por otra parte, el cuestionamiento de los paradigmas positivistas ha generado nuevos enfoques, 
que abordan la realidad social a través de diversas combinatorias de metodologías cualitativas y cuantitativas. De este modo, se ha cuestionado la noción de objetividad y neutralidad y se ha contribuido al esclarecimiento de los límites de la investigación(19).

El informe de la comisión Gulbenkian(20) sobre la 'reestructuración de las ciencias sociales' provee orientaciones enriquecidas por diversos análisis posteriores, como los de la UNESCO en 1999 y 2010(21,22). La pregunta recae sobre las formas más adecuadas para la construcción de conocimientos científico-sociales en la sociedad actual, reestructurando la forma de producción de conocimientos en el campo y reconectando la cultura de la ciencia y las humanidades(15).

Retomando el diálogo con otras ciencias, es pertinente recordar que el anhelo de conectar las ciencias tradicionales con las humanidades era parte de los primeros proyectos de la bioética, que veían en el diálogo interdisciplinario un asunto de relevancia ética tendiente a romper la aparente fractura secular entre ambos discursos(23).

La discusión se torna más compleja al tomar conciencia de que, en Latinoamérica, gran parte de las discusiones acerca de la ética de la investigación se liga a contextos sociales particulares, determinados por condiciones de carestía económica o psicosocial. El trabajo científico con seres humanos habitualmente se liga a poblaciones en situación de vulnerabilidad o en condiciones de asimetría respecto de la cultura dominante(24).

Compartimos con Achío(1) que el tipo de objeto de estudio en la investigación en ciencias sociales y las características comunes entres los/las investigadores y los/as sujetos de investigación producen problemas éticos diferentes a los de la investigación biomédica. Los efectos de una intervención social son menos tangibles, lo que dificulta reparar los daños o anticipar los riesgos potenciales de los/as sujetos participantes.

Un ejemplo es la Ley no 20.120, publicada en Chile el año 2006. En su primer artículo, limita la investigación científica al ámbito biomédico. La pregunta es si solo habría que pedir permiso a una persona cuando intervenimos su cuerpo y no cuando nos introducimos en sus deseos, sus procesos mentales, valores y, en definitiva, en su subjetividad(25).

Muchos de los aspectos propios de la subjetividad implicados en el daño físico o que conllevan malestar psicológico, o la posibilidad de iatrogenia, no pueden ser respondidos desde la biología. Tampoco desde una condensación entre la ética y la biología.

La pregunta que este artículo busca despejar recae sobre el interés que la norma ética y jurídica pone en las implicancias particulares de las ciencias sociales en el campo de la ética.

La decisión de comprender a la psicología como ciencia social responde a la necesidad de acotar, mediante un ejemplo, el extenso campo de las ciencias sociales y sus tópicos. Cabe señalar que la psicología constituye una disciplina particular, que establece nexos y códigos comunes tanto con las ciencias sociales como con las biomédicas. Desde este punto de vista, resulta relevante acceder a la regularidad y profundidad con que se consideran aspectos psicosociales en la redacción de las normas que regulan la investigación científica.

\section{Método}

Se abordó el presente estudio a través de una metodología mixta, propia del análisis de contenido, con un diseño cuantitativo y otro cualitativo.

Se utilizó un diseño cuantitativo no experimental, transeccional, de tipo exploratorio(26), que a través del uso de estadísticos simples mostró la frecuencia y porcentaje de aparición de vocablos pertenecientes al campo de la psicología en los documentos previamente seleccionados, lo que corresponde al criterio sintáctico del análisis de contenido(27), interesado en la morfología del texto a través de la búsqueda de la repetición de caracteres y palabras.

El diseño cualitativo utilizado fue de tipo emergente, a partir de un conjunto de procedimientos centrados en el criterio semántico del análisis de contenido(27), que busca el sentido de las palabras y el análisis de los temas que emergen de los documentos. 


\section{Clasificamos los documentos analizados en:}

1. Pautas y lineamientos éticos internacionales que regulan la investigación científica (declaraciones y tratados internacionales de ética, marcos éticos generales).

2. Normas jurídicas nacionales, que regulan el ejercicio de la investigación científica (Chile).

Siguiendo los planteamientos de Valles(28), la evidencia documental fue seleccionada a partir de dos criterios diferenciales: respecto de las normas éticas internacionales, se abordaron las normas con mayor amplitud de temas y mayor reconocimiento internacional.

En relación con las normas jurídicas chilenas, se seleccionaron los cuerpos legales (leyes, decretos, reglamentos) que regulan en forma general los procedimientos que en Chile deben ser realizados para generar investigación científica.

Conscientes de la naturaleza diversa de ambos tipos de documentos, nuestro interés recayó en explorar las distinciones de forma y fondo existentes en ellos. Se procedió recolectando y ordenando los documentos en forma cronológica y buscando las unidades de registro(29) a partir de indicadores de temas presentes en el material analizado (presencia o ausencia de normas específicas para la investigación en ciencias sociales).

La búsqueda fue orientada en torno a conceptos de la psicología, por ser esta una de las ciencias reconocidas por su aporte específico al campo de la ética de la investigación científica y por ser una disciplina representativa del campo intersticial entre las ciencias biomédicas y las ciencias sociales, en tanto hace confluir aspectos psicobiológicos y socioculturales.

Se procedió a revisar en cada documento la presencia o ausencia de normas específicas que regulan la investigación científica en ciencias sociales, utilizando las categorías Sí o No.

Posteriormente, fueron revisados los documentos a través de la herramienta de búsqueda de palabras del programa Microsoft Word 2007, siguiendo el criterio sintáctico del análisis de contenido.
Los vocablos y significados pesquisados fueron: psicología, psicológico/a, psiquismo, psíquico, psicosocial, comportamiento (en la acepción de ciencias del comportamiento), mental y mente.

A continuación, fue asignada una letra a cada vocablo, siendo distribuidas del modo descrito en tabla 1:

Tabla 1. Distribución de los vocablos y significados asociados a la psicología

\begin{tabular}{|l|l|}
\hline Letra a & $\begin{array}{l}\text { Presencia de palabras ligadas o derivadas de psi- } \\
\text { cología, entre ellas: psicológico/a, psíquico/a, } \\
\text { psiquismo, psique }\end{array}$ \\
\hline Letra b & Presencia de la palabra 'psicosocial' \\
\hline Letra c & $\begin{array}{l}\text { Palabras ligadas al concepto de comportamiento } \\
\text { (ciencias del), entre ellas: comportamental, cien- } \\
\text { cias del comportamiento. }\end{array}$ \\
\hline Letra d & $\begin{array}{l}\text { Palabras ligadas al concepto de lo mental. Entre } \\
\text { ellas: mente, mental, salud mental, daño mental }\end{array}$ \\
\hline
\end{tabular}

A continuación, se analizaron los datos en un nivel semántico, buscando comprender el sentido y uso de las palabras a través de los párrafos en que se presentan. En este caso, fue realizada una codificación abierta, con el objetivo de identificar elementos comunes a través de un criterio inductivo.

De los cinco cuerpos normativos internacionales seleccionados, encontramos que tres (60\%) abordan aspectos éticos en la investigación en ciencias sociales, siendo en todos los casos referencias generales, poco específicas o con escasas definiciones de los conceptos asociados.

De un total de 37.273 palabras revisadas en los códigos internacionales, solo 41 corresponden a los conceptos definidos por los investigadores como indicadores de la presencia de vocablos y significados de la psicología, comprendida como ciencia social, lo que constituye un $0,10 \%$ del total de palabras revisadas en este apartado.

El análisis semántico fue sistematizado en torno a dos de los principios fundamentales de la bioética científica, que como categorías inductivas sirvieron para organizar el material revisado. A saber, el principio de beneficencia/no maleficencia y el principio de autonomía. 


\section{Codificación}

A continuación, es presentada la categorización abierta que vincula la información incorporando la perspectiva crítica en el estudio(33).

1. Respeto del principio de autonomía: definido como la utilización de referencias asociadas a la libertad de las personas que participan en una investigación, para consentir o no las intervenciones que el/la investigador realiza.

1.1. Inclusión del consentimiento informado, comprendido como un proceso transversal y contextual (Pautas Éticas CIOMS):

"La obtención del consentimiento informado es un proceso que comienza con el contacto inicial con el potencial sujeto y continúa durante el estudio. Los investigadores solicitan el consentimiento mediante la información, repetición y explicación a los potenciales sujetos, respondiendo a sus preguntas a medida que surjan y asegurando que cada individuo entiende todos los procedimientos. Con esto, los investigadores manifiestan respeto por la dignidad y autonomia de las personas. Se debe dar a cada individuo el tiempo necesario para tomar una decisión, incluyendo tiempo para consultar a miembros de su

Tabla 2. Revisión de normas éticas internacionales familia u otros"(33).

1.2. Concepto de lo 'mental' o 'psicológico', asociado a la idea de disminución de la autonomía, minusvalía, inhabilidad o incapacidad para otorgar consentimiento (Declaración de Helsinki):

"La investigación en individuos que no son capaces fisica o mentalmente de otorgar consentimiento, por ejemplo, pacientes inconscientes, se puede realizar solo si la condición fisicalmental que impide otorgar el consentimiento informado es una característica necesaria de la población investigada"(31).

2. Respecto del principio de beneficencia/no maleficencia: corresponde a las referencias que se centran en la búsqueda activa de beneficios o la evitación activa de generar daño por parte de los/las investigadores.

2.1. Principio de beneficencia/no maleficencia, comprendido como una obligación ética de los/ las científicos/as (Informe Belmont):

"En toda investigación biomédica en sujetos humanos, el investigador debe garantizar que los beneficios potenciales y los riesgos estén razonablemente balanceados y que los riesgos hayan sido minimizados"(32).

\begin{tabular}{|c|c|c|c|c|c|c|}
\hline \multirow[t]{2}{*}{ Año } & \multirow[t]{2}{*}{ Nombre del texto } & \multicolumn{2}{|c|}{$\begin{array}{l}\text { Presencia de normas éticas } \\
\text { específicas para las ciencias } \\
\text { sociales }\end{array}$} & \multirow[t]{2}{*}{$\begin{array}{l}\text { Número de } \\
\text { palabras del } \\
\text { texto }\end{array}$} & \multirow{2}{*}{$\begin{array}{l}\text { Frecuencia } \\
\text { de aparición } \\
\text { de vocablos } \\
\text { psicológicos }\end{array}$} & \multirow{2}{*}{$\begin{array}{l}\text { Porcentaje de } \\
\text { uso de voca- } \\
\text { blos psicoló- } \\
\text { gicos }\end{array}$} \\
\hline & & Sí & No & & & \\
\hline 1947 & $\begin{array}{l}\text { Código de Nüren- } \\
\text { berg (30) }\end{array}$ & & $\mathrm{X}$ & 590 & 2 & $0,33 \%$ \\
\hline 1964 & $\begin{array}{l}\text { Declaración de Hel- } \\
\text { sinki (31) }\end{array}$ & & $\mathrm{X}$ & 2.254 & 3 & $0,13 \%$ \\
\hline 1979 & $\begin{array}{l}\text { El Informe Belmont } \\
\text { (32) }\end{array}$ & $\mathrm{X}$ & & 5.723 & 6 & $0,10 \%$ \\
\hline 2002 & Pautas CIOMS (33) & $\mathrm{X}$ & & 24.502 & 28 & $0,11 \%$ \\
\hline 2005 & $\begin{array}{l}\text { Declaración Univer- } \\
\text { sal sobre Bioética y } \\
\text { Derechos Humanos } \\
\text { (34) }\end{array}$ & $\mathrm{X}$ & & 4.204 & 2 & $0,04 \%$ \\
\hline Totales & & 3 & 2 & 37.273 & 41 & $0,10 \%$ \\
\hline
\end{tabular}


2.2. Concepto de lo 'mental' o 'psicológico', asociado a la posibilidad de generar daño o perjuicio, basado en la investigación/intervención (Declaración de Helsinki):

"Deben tomarse toda clase de precauciones para resguardar la intimidad de la persona que participa en la investigación y confidencialidad de su información personal, y para reducir al minimo las consecuencias de la investigación sobre su integridad fisica o mental"(31).

Del análisis semántico de los textos se desprende una evidente ausencia de conceptos asociados a la salud mental y una dificultad para definirlos, delimitarlos e integrarlos en el concierto de las demás normas.

De modo similar que en la tabla 2, de las cinco normas jurídicas revisadas, dos (40\%) abordan aspectos éticos en el área específica de las ciencias sociales, siendo referencias generales o con escasas definiciones de los conceptos asociados.

De un total de 41.372 palabras revisadas en las normas jurídicas chilenas, solo nueve palabras corresponden a los conceptos definidos por los investigadores como indicadores de la presencia de vocablos y significados de la psicología, lo que constituye un $0,02 \%$ del total de palabras revisadas.

\section{Codificación}

Se presenta a continuación la codificación abierta realizada con el material jurídico examinado:

1. Respecto del principio de autonomía: corresponde a la utilización de referencias asociadas a la libertad de las personas que participan en una investigación, para consentir o no las intervenciones que el/la investigador/a realiza. Se agrega en este caso la protección jurídica que puede implicar el respeto a este principio.

Relevancia del concepto de consentimiento informado (Ley 20.120):

"Artículo 11. Toda investigación cientifica en un ser humano deberá contar con su consentimiento previo, expreso, libre e informado o, en su defecto, el de aquel que deba suplir su voluntad en conformidad con la ley"(38).

Tabla 3. Revisión de normas jurídicas chilenas

\begin{tabular}{|c|c|c|c|c|c|c|}
\hline \multirow[t]{2}{*}{ Año } & \multirow[t]{2}{*}{ Nombre del texto } & \multicolumn{2}{|c|}{$\begin{array}{l}\text { Presencia de normas éticas } \\
\text { específicas para las ciencias } \\
\text { sociales }\end{array}$} & \multirow[t]{2}{*}{$\begin{array}{ll}\text { Número de } \\
\text { palabras del } \\
\text { texto }\end{array}$} & \multirow{2}{*}{$\begin{array}{l}\text { Frecuencia } \\
\text { de aparición } \\
\text { de vocablos } \\
\text { psicológicos }\end{array}$} & \multirow{2}{*}{$\begin{array}{l}\text { Porcentaje } \\
\text { de uso de } \\
\text { vocablos psi- } \\
\text { cológicos }\end{array}$} \\
\hline & & Sí & No & & & \\
\hline 1980 & $\begin{array}{l}\text { Constitución Política de la Re- } \\
\text { pública de Chile (35) }\end{array}$ & & $\mathrm{X}$ & 25.835 & 2 & $0,007 \%$ \\
\hline 1999 & $\begin{array}{l}\text { Ley } 19.628 \text { Protección de datos } \\
\text { de carácter personal ( } 36 \text { ) }\end{array}$ & & $\mathrm{X}$ & 4.109 & 1 & $0,024 \%$ \\
\hline 2001 & $\begin{array}{l}\text { Norma Técnica no } 57 \text { Regula- } \\
\text { ción de la ejecución de ensayos } \\
\text { clínicos que utilizan productos } \\
\text { farmacéuticos en seres huma- } \\
\text { nos (37) }\end{array}$ & & $\mathrm{X}$ & 6.945 & 0 & $0 \%$ \\
\hline 2006 & $\begin{array}{l}\text { Ley } \mathrm{n}^{\circ} 20.120 \text { Sobre la inves- } \\
\text { tigación científica en el ser hu- } \\
\text { mano, su genoma y prohíbe la } \\
\text { clonación humana(38) }\end{array}$ & $\mathrm{X}$ & & 1.336 & 2 & $0,149 \%$ \\
\hline 2011 & $\begin{array}{l}\text { Reglamento de Ley } 20.120 \\
\text { (39) }\end{array}$ & $\mathrm{X}$ & & 3.147 & 4 & $0,127 \%$ \\
\hline Totales & & 2 & & 41.372 & 9 & $0,021 \%$ \\
\hline
\end{tabular}


1.2. Concepto de 'lo mental' o 'psicológico', asociado a la idea de disminución, minusvalía, daño o limitación jurídica (Constitución Política de la República de Chile):

"Articulo 49. No 7: Son atribuciones exclusivas del Senado: Declarar la inhabilidad del Presidente de la República o del Presidente electo cuando un impedimento físico o mental lo inhabilite para el ejercicio de sus funciones"(35).

1.3. Concepción 'aflictiva' de la enfermedad mental (Ley 20.120):

“Artículo 12. Párrafo 20: Cuando la investigación incluya el otorgamiento y prestaciones o acciones de salud a personas que padezcan enfermedades mentales, estas podrán dar su consentimiento en la forma prevista en el decreto supremo No 570 del Ministerio de Salud, que aprueba el reglamento para la internación de las personas con enfermedades mentales y sobre los establecimientos que la proporcionan"(38).

2. Respecto del principio de beneficencia/no maleficencia: corresponde a la búsqueda activa de beneficios o la evitación activa de generar daño por parte de los/las investigadores/as, agregándosele la protección jurídica que garantiza la ley.

2.1 Protección jurídica a la integridad física y psíquica (Ley 20.120):

"Toda investigación cientifica en seres humanos que implique algún tipo de intervención física o psiquica deberá ser realizada siempre por profesionales idóneos en la materia, justificarse en su objetivo y metodología y ajustarse en todo a lo dispuesto en esta ley"(38).

\section{Conclusiones}

La búsqueda de referencias a la psicología en los cuerpos normativos incluidos impone consideraciones que llevan a distinguir la naturaleza diversa de las normas abordadas, tanto respecto de su origen como en cuanto a su propósito.

La finalidad de las normas éticas internacionales en investigación científica consiste en resguardar el cumplimiento de principios que garanticen el correcto proceder de los y las investigadores/as en contextos que involucren trabajar con seres humanos. Los principios contenidos en estos códigos fundamentan sus prescripciones y encuentran un alto nivel de consenso en el concierto internacional; sin embargo, no tienen efecto de obligatoriedad legal ni se asocian a consecuencias punitivas. Solo se instituyen como orientación del trabajo científico.

En el caso de las normas jurídicas, el propósito fundamental es mandar, prohibir o permitir los comportamientos de los y las investigadores/as, relacionados con la investigación científica, en el marco de los sistemas de garantía y protección del cumplimiento del derecho chileno. Recae sobre el Estado la función de garante del progreso científico, en el marco del respeto a los derechos humanos consagrado en la Constitución de la República y en la adscripción a los principios éticos internacionales ratificados por la ley. Es relevante considerar la profunda imbricación entre ética y Derecho.

En la mayoría de los códigos y leyes se hacen alcances poco específicos a los estudios o intervenciones psicológicas, evidenciándose una total ausencia de definición o delimitación de estos conceptos, y dándose por entendidos en el contexto y alcance global de la norma.

Se comprueba así que las regulaciones éticas y legales ligadas a la investigación han priorizado, como foco de interés, la relevancia que adquiere la investigación biomédica con seres humanos y las consecuencias que de esta se desprenden, dejando en segundo plano las especificidades y dilemáticas éticas propias de las ciencias sociales.

La revisión del caso específico de la psicología muestra una restringida referencia, asociada, en la mayoría de los casos, a conceptos ligados a la minusvalía, discapacidad, daño o enfermedad psicológica/mental.

Merece ser destacada la connotación proteccionista que la ley otorga a situaciones en las que se vea involucrada la posibilidad de daño físico o psicológico en el contexto de la investigación, sindicando a los/las científicos/as como los portadores de la responsabilidad social de la ciencia. 
No obstante, se aprecia, en términos generales, que los vocablos y significados se inscriben en un contexto amplio 'principalista', en el cual la ética de la investigación se apoya fundamentalmente en dos principios propios de la bioética: el de autonomía y el de beneficencia/no maleficencia.

Respecto del principio de autonomía, ha sido encontrado en ambos tipos de cuerpos normativos, siendo el consentimiento informado el núcleo de los esfuerzos de los/las investigadores/as por respetar la libertad que los individuos tienen de decidir sobre su participación en una investigación científica.

El principio de beneficencia/no maleficencia se traduce en normas que destacan el cuidado que los/las investigadores/as deben poner en no ejercer daño físico o psicológico en seres humanos, considerando el respeto a los derechos humanos y las garantías que el Estado debe a la protección de las personas.

La baja frecuencia de aparición de conceptos psicológicos no es significativamente diferente si comparamos los resultados de la revisión de los códigos éticos internacionales y las leyes chilenas, lo que da cuenta del similar estatuto que se otorga a la psicología en el contexto de ambos tipos de norma. Evidentemente, este dato refuerza la idea de que la psicología no se constituye como un insumo de las preocupaciones éticas de quienes desarrollan las normativas, salvo en los casos en que sirve para especificar los límites de algunos principios y normas.

La mayoría de los códigos separa los intereses y objetivos de la bioética del ámbito de los intereses psicológicos o sociales. Existe una tendencia a referirse a lo 'mental' o 'psicológico' como algo completamente ajeno a lo biológico.

Los resultados demuestran que existe una ausencia evidente de conceptos ligados a una función 'positiva' de la salud mental, la promoción-prevención o los avances científicos en estas materias.

Considerando las importantes carencias de normas, definiciones y procedimientos que orientan el proceder ético en la investigación en ciencias sociales, nos parece de gran relevancia retomar las voces e inquietudes que apuntan a la generación de un cuerpo ético-jurídico particular para este ámbito. No es desdeñable que, si las cuestiones éticas han de inscribirse en coordenadas sociales contextuales, la ética misma puede ser concebida como una 'cuestión social', susceptible de ser estudiada desde los enfoques comprensivos de las ciencias sociales.

Los conflictos y dilemas éticos que se desprenden de los estudios científicos son diferenciales, sean o no empíricos, cualitativos o cuantitativos. En el caso de las ciencias biomédicas, se generan condiciones que llaman a la consideración ética en investigaciones que implican el uso del material biológico de seres humanos. En el caso de las ciencias sociales, existen particularidades éticas, habitualmente asociadas al tratamiento ético del material obtenido a partir de los llamados 'datos sensibles', como es el caso de la orientación sexual, las opiniones políticas, religiosas o cualquier tema que implique la intimidad subjetiva de la persona que presta su consentimiento para la investigación.

La bioética fue originalmente pensada como una disciplina asociada a las 'ciencias de la vida', en su acepción más amplia, de modo que es posible pensar en integrar a esta concepción las ciencias sociales. El desafío está expuesto y es actualmente tema de controversia. Es requerida la voluntad política de los/las legisladores/as y la responsabilidad social de los/as científicos/as para que converjan nuevos espacios de reflexión ética que incluyan las dimensiones sociales y biológicas del ser humano en cuerpos normativos coherentes y ecológicamente sensibles. 
Revisión de pautas nacionales e internacionales en ética de la investigación científica - María Inés Winkler y Antonio Letelier

\section{Referencias}

1. Achío M. Ética de la investigación en Ciencias Sociales: Repensando temas viejos. Perspectivas Bioéticas [en línea] 2006, II semestre [acceso 12 enero 2012]; 15(15). Disponible en: http://es.scribd.com/doc/52875437/Etica-de-la-investigacion-en-ciencias-sociales

2. Drane J. La ética como carácter y la investigación médica. Acta Bioethica [en línea] 2004 [acceso 25 noviembre 2011]; 10(1): Disponible en: http:/www.scielo.cl/scielo.php?pid=S1726-569X2004000100003\&script=sci_arttext

3. Kotow M. Tensiones retóricas y semánticas en ética de la investigación. Cad Saúde Pública [en línea] 2007, Oct. [acceso 19 mar 2012]; 23(10). Disponible en: http://www.scielosp.org/pdf/csp/v23n10/14.pdf

4. Gaudlitz M. Reflexiones sobre los principios éticos en investigación biomédica en seres humanos. Rev Chil Enf Respir [en línea] 2008 [acceso 19 mar 2012]; 24(2): 138-142. Disponible en: http://www.scielo.cl/scielo.php?pid=S071773482008000200008\&script=sci_arttext

5. Sotomayor M.A. Regulación de la investigación biomédica en Chile. Acta Bioethica [en línea] 2008 [acceso 28 abril 2012]; 14(1): 79-89. Disponible en: http://www.scielo.cl/scielo.php?script=sci_arttext\&pid=S1726-569X2008000100011

6. Lolas F. Bioética en América Latina. Una década de evolución [en línea] Santiago de Chile: Centro Interdisciplinario de Estudios en Bioética, Universidad de Chile, Programa de Bioética OPS/OMS; 2010. [acceso 20 mayo 2012] Disponible en: http://www.uchile.cl/portal/investigacion/centro-interdisciplinario-de-estudios-en-bioetica/publicaciones/76704/libros

7. Mora E. La revolución científico-técnica en la educación de los tecnólogos de salud. ACIMED [en línea] 2008 [acceso el 24 abril 2012]; 18(5). Disponible en: http://scielo.sld.cu/scielo.php?script=sci_arttext\&pid=S1024-94352008001100003

8. Rovaletti L. La evaluación ética en las Ciencias Humanas y/o Sociales. La investigación científica: entre la Libertad y la Responsabilidad. Acta Bioethica [en línea] 2006 [acceso 21 marzo 2012]; 12(2): 243-250. Disponible en: http://www. scielo.cl/scielo.php?pid=S1726-569X2006000200013\&script=sci_arttext

9. Maliandi R. Ética convergente. Fenomenología de la conflictividad. Tomo I/III. 1ª ed. Buenos Aires: Las Cuarenta; 2010.

10. Unesco.org. Declaración sobre la ciencia y el uso del saber cientifico [en línea] 1999 [acceso 25 abril 2012]. Disponible en: http://www.unesco.org/science/wcs/esp/declaracion_s.htm

11. Laufer M. La dimensión ética en la ciencia y la tecnología. Interciencia [en línea] 2006 [acceso 25 abril 2012]; 31(7): 473-475. Disponible en: http://redalyc.uaemex.mx/pdf/339/33911801.pdf

12. Emanuel E. ¿QQué hace que la investigación clínica sea ética? Siete requisitos éticos. En: Pellegrini A, Macklin R, (eds). Investigación en Sujetos Humanos: Experiencia Internacional. Santiago de Chile: Programa Regional de Bioética OPS/ OMS; 1999: 33-46.

13. Aréchiga H. Los aspectos éticos de la ciencia moderna. En: Aluja M, Birke, A, (coords.) El papel de la ética en la Investigación Cientifica y la Educación superior. 2a ed. México: FCE, Academia Mexicana de Ciencias; 2004: 41-65.

14. Garretón M. Las ciencias sociales en Chile. Institucionalización, ruptura y renacimiento. [en línea] 2005 [acceso 25 abril 2012]. Disponible en: http://www.manuelantoniogarreton.cl/documentos/07_08_06/sociales.pdf

15. Ramos-Zincke C, Canales A, Palestini S. El campo de las ciencias sociales en Chile. ¿Convergencia disciplinar en la construcción del objeto de estudio? Cinta de Moebio [en línea] 2008 [acceso 22 mayo 2012]; 33: 171-194. Disponible en: http://www.scielo.cl/scielo.php?pid=S0717-554X2008000300002\&script=sci_arttext

16. Sánchez A. Ética de la Intervención Social. $1^{\mathrm{a}}$ ed. Barcelona: Paidós; 1999.

17. Neira J. Ciencias sociales y salud. Pre-textos para el debate. Revista Austral de Ciencias Sociales [en línea] 2001 [acceso 24 mayo 2012]; 5: 127-138. Disponible en: http://mingaonline.uach.cl/scielo.php?pid=S071817952001000100011\& script=sci_arttext

18. Erazo M. Rigor científico en las prácticas de investigación cualitativa. Ciencia, Docencia Tecnología [en línea] 2011 [acceso 19 mayo 2012]; 42: 107-136. Disponible en: http://www.scielo.org.ar/scielo.php?pid=S18511716201100010 0004\&script=sci_arttext

19. Lira E. Notas sobre ética, investigación social y derechos humanos. En: Bioética en Investigación en Ciencias Sociales. 3er taller organizado por el comité asesor de bioética de Fondecyt- CONICYT [en línea]. Santiago de Chile: 2008 [acceso 21 mayo 2012]: 107-136. Disponible en: http://www.conicyt.cl/573/articles-31324_recurso_2.pdf

20. Wallerstein Inmanuel (coordinador). Comision Gulbenkian para la reestructuración de las ciencias sociales. México DF: Siglo XXI Editores.

21. Unesco.org. Declaración sobre la ciencia y el uso del saber científico. [en línea]. Budapest; 1999 [acceso 30 junio 2012]. Disponible en: http://www.unesco.org/science/wcs/esp/declaracion_s.htm

22. Unesco.org. Informe sobre las ciencias sociales en el mundo. Las brechas del conocimiento. [en línea]. México D.F: foroconsultivo.org.mx; 2010; [acceso 30 junio 2012]. Disponible en: http://www.foroconsultivo.org.mx/libros_editados/ informe_sobre_las_ciencias_sociales_en_el_mundo.pdf 
23. Wilches A. La propuesta bioética de Van Rensselaer Potter, cuatro décadas después. Opción [en línea] 2011 [acceso 24 mayo 2012]; 66: 70-84. Disponible en: http://revistas.luz.edu.ve/index.php/op/article/viewFile/10060/9734

24. Flores J. Psicología, Ética y Proceso de Globalización en América Latina. Rev. Internacional de Psicología [en línea] 2001 [acceso 30 junio 2012]; 2(1). Disponible en: http://psicologiarevista.99k.org/Psicologia,\%20Etica\%20y\%20Proceso\%20de\%20Globalizacion\%20en\%20America\%20Latina.pdf

25. Michaud P. Introducción. En: Bioética en Investigación en Ciencias Sociales. $3^{\text {er }}$ taller organizado por el comité asesor de bioética de Fondecyt-CONICYT. Santiago de Chile [en línea]; 2008: 11-16. [acceso 23 julio 2012] Disponible en: http://www.conicyt.cl/573/articles-31324_recurso_2.pdf

26. Hernández R, Fernández C, Baptista P. Metodología de la Investigación. 4a ed. México: Mc Graw-Hill; 2006.

27. Amezcua M, Gálvez A. Los modos de análisis en investigación cualitativa en salud: perspectiva crítica y reflexiones en voz alta. Rev. Esp. Salud Pública [en línea] 2002 [acceso 23 abril 2012]; 76(5): 423-436. Disponible en: http://scielo. isciii.es/scielo.php?pid=s1135-57272002000500005\&script=sci_arttext

28. Valles M. Técnicas Cualitativas de Investigación Social. Reflexión Metodológica y Práctica Profesional [libro electrónico]. Madrid: Síntesis; 1999 [acceso 09 julio 2012]. Disponible en: http://es.scribd.com/doc/53039790/Valles-Miguel-Tecnicas-cualitativas-de-investigacion-social-Reflexion-metodologica-y-practica-profesional

29. Cáceres P. Análisis Cualitativo de Contenido: Una alternativa metodológica alcanzable. Rev. de la Escuela de Psicología. Fac. de Filosofía y Educ. [en línea] 2003 [acceso 09 julio 2012]; II: 53-82]. Disponible en: http://www.psicoperspectivas.cl/index.php/psicoperspectivas/article/viewFile/3/3

30. Tribunal Internacional de Nürenberg: Código de Nürenberg, 20 agosto, 1947. [acceso 26 abril 2012] Disponible en: http://www.bioeticanet.info/documentos/Nuremberg.pdf

31. Asamblea Médica Mundial: Declaración de Helsinki. Recomendaciones para orientar a los médicos en la investigación biomédica con seres humanos. Adoptadas por la 18a Asamblea Médica Mundial Helsinki. Finlandia (Junio, 1964) y enmendadas por la 29a Asamblea Médica Mundial. Tokio(Octubre, 1975), por la 35a Asamblea Médica Mundial. Venecia (Octubre, 1983) y por la 41ª Asamblea Médica Mundial. Hong-Kong (Septiembre, 1989). [acceso 26 abril 2012] Disponible en: http://www.uchile.cl/portal/investigacion/centro-interdisciplinario-de-estudios-en-bioetica/documentos/76030/declaracion-de-helsinki-de-la-asociacion-medica-mundial

32. Comisión Nacional para la protección de los sujetos humanos de investigación biomédica y del comportamiento. U.S.A. El Informe Belmont. Principios y guías éticos para la protección de los sujetos humanos de investigación; 18 abril, 1979. [acceso 26 Abril 2012] Disponible en: http://www.pcb.ub.edu/bioeticaidret/archivos/norm/InformeBelmont. pdf

33. CIOMS. Pautas éticas internacionales para la investigación biomédica en seres humanos. Ginebra: Consejo de las Ciencias Médicas (CIOMS), en colaboración con la Organización Mundial de la Salud; 2002. [acceso 26 abril 2012] Disponible en: http://www.cioms.ch/publications/guidelines/pautas_eticas_internacionales.htm

34. Unesco.org. Declaración Universal sobre Bioética y Derechos Humanos. [en línea] 2006; [acceso 30 junio 2012]. Disponible en: http://unesdoc.unesco.org/images/0014/001461/146180s.pdf

35. Chile. Ministerio Secretaría General de la Presidencia. Constitución Política de la República de Chile; sept. 17-2005. [acceso 26 abril 2012] Disponible en: http://www.minsegpres.gob.cl/wp-content/uploads/2011/01/Constituci\%C3\%B3nPol\%C3\%ADtica.pdf

36. Chile. Ministerio Secretaría General de la Presidencia. Ley 19.628 Sobre protección de la vida privada; agosto 17, 1999. [acceso 26 abril 2012] Disponible en: http://www.leychile.cl/Navegar?idNorma=141599

37. Chile. Ministerio de Salud. Norma Técnica $N^{\circ} 57$. Regulación de la ejecución de ensayos clínicos que utilizan productos farmacéuticos en seres humanos, junio 4, 2001. [acceso 26 abril 2012]. Disponible en: http://www.ispch. cl/sites/default/files/Norma\%20Tecnica\%2057.pdf

38. Chile. Ministerio de Salud. Ley 20.120 Sobre la investigación cientifica en el ser humano, su genoma, y prohíbe la clonación humana, septiembre 22, 2006. [acceso 27 abril 2012] Disponible en: http://www.huap.cl/docs/marconormativo4.pdf

39. Chile. Ministerio de Salud. Decreto 114 Aprueba Reglamento de la Ley No 20.120 Sobre la investigación cientifica en el ser humano, su genoma, y prohíbe la clonación humana, noviembre 19, 2011. [acceso 20 junio 2012]. Disponible en: http:// legislacion-oficial.vlex.cl/vid/humano-genoma-prohibe-clonacion-humana-333813034

Recibido: 12 de mayo de 2012

Aceptado: 1 de septiembre de 2012 